\title{
A Study on the Small Organic Cities in China Combining PCA and CNN
}

\author{
Mengdie Xu, Zhiwei Yang ${ }^{(D)}$, Zhiting Li, Die Liu, and Tan Yang \\ Chongqing College of Humanities, Science \& Technology, School of Architecture and Design, Chongqing 401524, China \\ Correspondence should be addressed to Zhiwei Yang; xczx@cqrk.edu.cn
}

Received 26 September 2021; Revised 26 October 2021; Accepted 30 October 2021; Published 17 November 2021

Academic Editor: Fazlullah Khan

Copyright ( $\odot 2021$ Mengdie Xu et al. This is an open access article distributed under the Creative Commons Attribution License, which permits unrestricted use, distribution, and reproduction in any medium, provided the original work is properly cited.

\begin{abstract}
Background. Organic development is one of the evolutionary development models for cities, and it can facilitate cities to continually adapt to their surroundings and therefore adjust their development. Former research is mostly focused on cities and environmental changes, and research on the organic development of cities in various conditions is rather scarce. Purpose. By creating an evaluation index system for organic cities, this paper has established a multifactor evaluation model and improved the evaluation methodologies for organic cities. Methods. The authors have come up with a method that combines PCA and CNN, established an optimized model to calculate the organic cities, verified the accuracy of the evaluation index system for organic cities, and finally concluded that urban organic development is mostly associated with the aspects of the natural environment, economic basis, land use, and population scale of the city. Case Study. In addition, the examples of Bishan District, Chongqing, and Jiangyin, Jiangsu Province, are simulated and compared, and an accurate prediction for the organic development of small cities in China is given. Conclusion. The evaluation index system for organic cities devised by this paper can be applied effectively to evaluate the organic city of cities, and the neural network prediction model for organic cities designed by this paper can provide references for further research on organic cities.
\end{abstract}

\section{Introduction}

Urban development evaluation is defined as the evaluation of a city in the process of urban development. On the one hand, it can be used as the guidance for the urban development of the next phase, and on the other hand, it can be referred to as the standard for similar cities' development. Common evaluation includes sustainability, ecological construction, green construction, low-carbon construction, and urban renewal. These often correspond to a certain field related to urban development; for example, the urban sustainable development evaluation index often corresponds to resource allocation, economic development, environmental protection, and social development [1-3]. The ecological city (green city) evaluation index is connected with resource-saving, eco-friendly character, and economic sustainability [4-8]. Urban renewal evaluation means evaluating the first, middle, and final phases of urban renewal [9-12]. The evaluation for urban development mentioned above sets most store by the changes of urban development and is focused on one aspect of urban development. Although it can provide some scientific guidance for the process of urban development, the drawback is obvious in that it fails to cover the overall situation of urban development.

At present, the commonly used evaluation methods are based on the establishment of the evaluation index system using the Delphi method, analytic hierarchy process, grey relational analysis, principal component analysis, and so on to determine the index weights and then calculate the results with the weighted means of the indices [13-17]. After setting a systematic and scientific index system and reasonable index weights, these methods can evaluate the weighted means of various evaluation indices and therefore indicate the development level and abilities of a city in a specific region more comprehensively and scientifically. However, the problems of these evaluation methods cannot be ignored; the 
results can only provide the rankings of the evaluated targets in most cases but cannot explain why evaluated targets with lower rankings take up such rankings and cannot provide clearer references to optimize the adjustment of evaluated targets. On top of that, these methods choose specific and limited indices and overlook the fact that the city is a complex megasystem, and many factors contribute to urban development in a nonlinear manner.

To address the problems mentioned previously, this paper comes up with a comprehensive evaluation for urban development combining the principal component analysis (PCA) and convolutional neural network (CNN), that is, the evaluation for the urban organic city. The concept of organic cities emphasizes that the urban system itself is structural and active like an organism, and the inner motive of urban development is featured with a virtuous cycle and can create an environment that can provide a healthy life for the citizens to the greatest extent [18]. This research applies PCA to establish the evaluation index system for organic cities, based on which the convolutional neural network is used to extract eigenvalues, and ResNet (Residual Network) is further utilized to establish the optimized model for urban organic feature calculation.

The major contributions of this paper are as follows:

(1) This research applies the principal component analysis (PCA) to create an evaluation index system for organic cities and improves the evaluation methods for the urban organic cities. This research establishes an evaluation system, which can make an objective evaluation of the existence of an organic city for urban construction.

(2) In addition, the research comes up with a method combining PCA and the convolutional neural network's residual network (ResNet), and as the network layer deepens, it introduces the residual connection to effectively address the problem of the training's increasing errors caused by too many layers in the real network training and ultimately improves the accuracy of the prediction for the organic cities.

(3) This research establishes an optimized model for calculating urban organic cities and chooses Bishan and Jiangyin for the case study.

The rest of the paper is organized according to the following pattern.

In Section 2, the establishment of the evaluation index system for organic cities is discussed. In Section 3, the PCA evaluation method is discussed. In Section 4, the case tests for organic cities are conducted. In Section 5, the experiments are conducted on organic cities that apply the convolutional neural network's residual network ResNet. In Section 6, the CNN analysis results of geographic and ecological indices, urban industrial and economic structure, and population development are discussed. In Section 7, the comparison analysis is conducted, and then the whole paper is concluded in Section 8 .

\section{Related Work}

In Section 2, the evaluation of the index system for organic cities is discussed. Also, the establishment of the evaluation index system that consists of five parts is discussed.

2.1. The Evaluation of Index System for Organic Cities. Following are the major steps for the evaluation of index system for organic cities:

(1) The nature of organic cities is analyzed. The research should analyze both the nature of the chosen city and the focus of the construction and, with relevant analysis, determine the indices and their weights in the evaluation index system.

(2) The index system is established. The research aims to establish an index system that can be applied for general situations, but in actual application, for different features of different cities, the indices can be modified and improved.

(3) The evaluation method is determined. The research chooses the principal component analysis to truly indicate the nature and construction focus of the city (as shown in Figure 1).

The steps to establish the evaluation index system for organic cities are presented in Figure 1.

2.2. The Establishment of the Evaluation Index System. The whole evaluation index system consists of 5 parts (as shown in Figure 2).

(i) Target A: the composite index for organic cities indicates the level of urban organic development.

(ii) System B: the target is further divided into 4 systems according to the constituent systems of the idea of organic cities.

(iii) Subsystem C: the systems are further divided.

(iv) Indicator D: the constituent factors of the idea of organic cities are connected with corresponding systems and subsystems (as shown in Table 1).

\section{Method}

In Section 3, the PCA evaluation method is discussed, which transforms linearly collected variables into new ones and then sequences the new variables in the order of decreasing variances.

3.1. The Application of the PCA Evaluation Method. PCA transforms linearly collected variables to new ones and then sequences the new variables in the order of decreasing variances. In the mathematical transformation, the total variance keeps constant, then the first variable will have the greatest variance, being the first principal component, and 


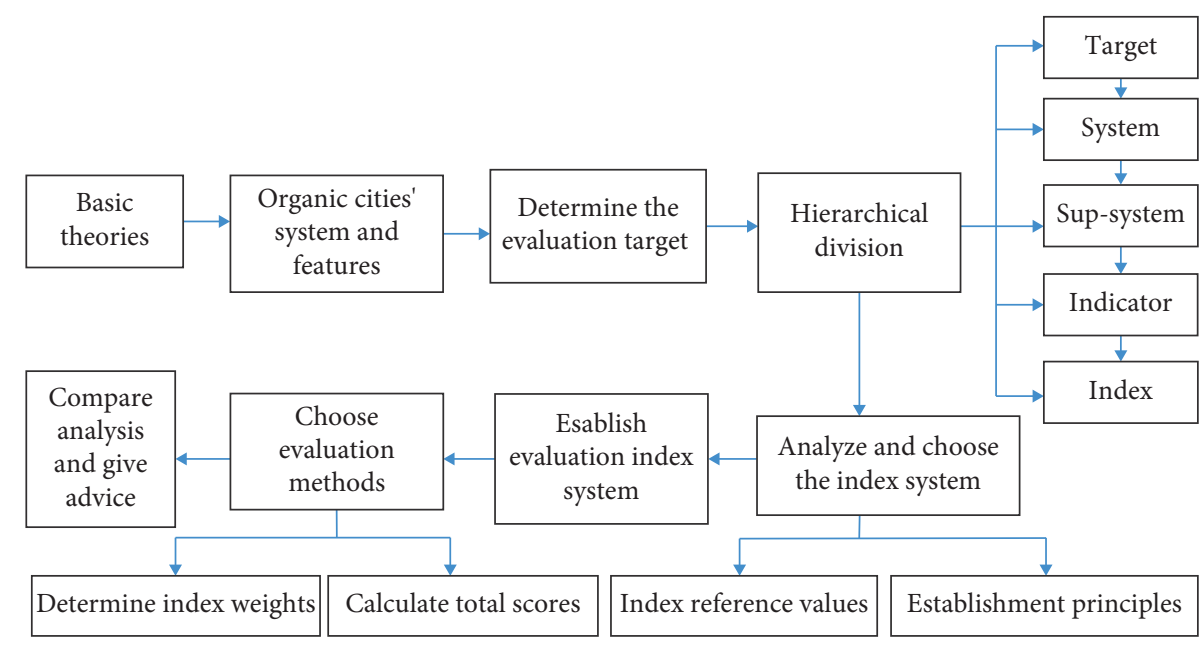

FIgURE 1: Construction steps of organic city evaluation index system.

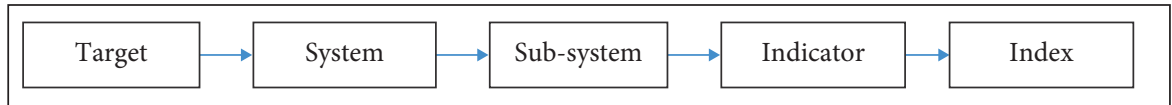

FIGURE 2: Hierarchical graph of the evaluation system.

by the same token, each variable will have one principal component. PCA is a method that reduces dimension.

3.1.1. Standardizing Evaluation Indices. Suppose that there are $k$ indices and $n$ samples and the original data matrix can be obtained (as shown in Table 2).

Then, corresponding standardized data for each index $x_{i j}$ are as follows:

$$
x_{i j}=\frac{X_{i j}}{\sum_{i=1}^{n} X_{i j}},
$$

where $i=1,2, \ldots, n, j=1,2, \ldots, k$. After the standardization, the value for each index can be limited to $[0,1]$.

3.1.2. Determining the Weights of Evaluation Indices. The SPSS software is applied to analyze the main components of the collected data, which correspond to each index in the index system, and the explained variance rate and score coefficient matrix of each principal component are presented in Table 3 (as shown in Table 3).

From the table, it can be seen that the former 5 principal component eigenvalues are greater than 1 , their contribution rates are greater than $85 \%$, and accumulated variance contribution rates reach up to $95.758 \%$, which can cover the information needed for evaluation more comprehensively. Summing the products of 41 indices' coefficients in 5 principal components and the variance contribution rates of principal components and after the process of standardization, the weighted vector for each evaluation factor is as follows:

$$
W=\sum_{a=1}^{m} g_{q} \cdot a_{q i},
$$

where $g_{q}$ is the variance contribution rate of $q$ principal component, $a_{q i}$ is the score coefficient of $i$ factor in $q$ principal component, and $w$ is the weighted vector. The weighted value can be obtained then.

\section{Case Tests for Organic Cities}

In this section, the small cities are selected for the case study. The table of urban organic composite index evaluation is presented in Table 4, and then the organic composite index and scores of each system and index are calculated as shown in Table 5. The statistical results of the actual indices of the urban growth boundary, urban scale, urban economy, and urban environment are presented in Table 6 . The analysis of the urban organic composite index is discussed in the following.

4.1. Choosing the Cases. This paper only researches small cities in China. According to Announcement Concerning the Adjustment of Urban Scale Classification Criteria published by the State Council in November 2014, the small cities in this research are cities (urban districts) whose population is smaller than 500,000. Bishan District, Chongqing, is chosen as the research target in this paper.

4.2. The Establishment of Urban Organic Indices. Chongqing's Annals of Statistics, Bishan's Annals of Statistics, statistical communique of people's economic and 
TABLE 1: Index E: this paper chooses various indices that correspond with the indicator.

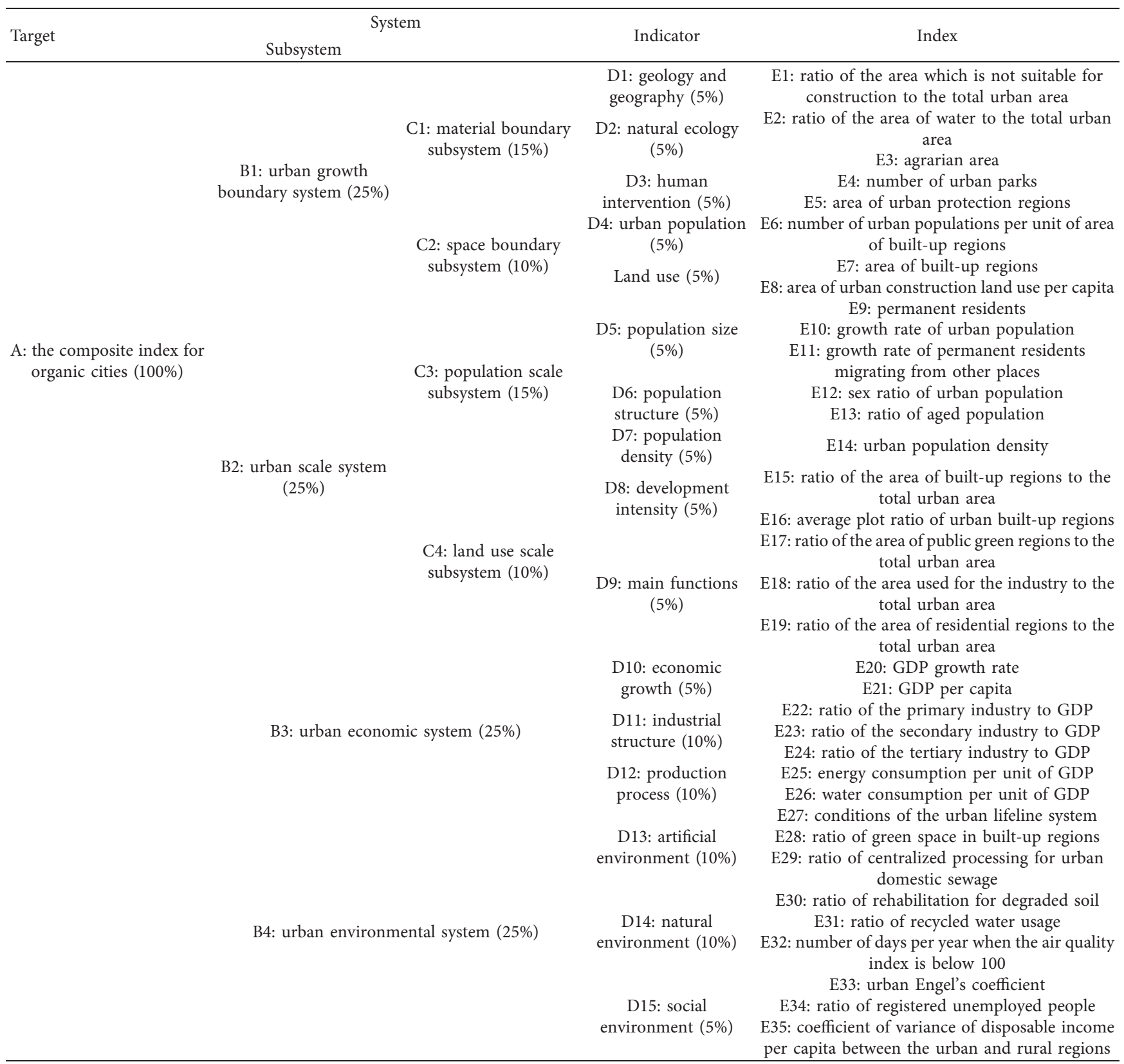

TABLE 2: The original data matrix.

\begin{tabular}{lcccc}
\hline Sample & $\mathrm{A} 1$ & $\mathrm{~A} 2$ & $\ldots$ & \\
\hline S1 & $\mathrm{X} 11$ & $\mathrm{X} 11$ & $\ldots$ & \\
$\mathrm{S} 2$ & $\mathrm{X} 21$ & $\mathrm{X}$ & $\ldots$ & $\mathrm{X} 1 k$ \\
Sn & $\mathrm{X} n 1$ & $\mathrm{X} n 2$ & $\ldots$ & $\mathrm{X} 2 k$ \\
\hline
\end{tabular}

social development, the eleventh and twelfth 5-year plans, and so on are referred to for acquiring 350 data in total from 2011 to 2020 . Using the evaluation index system for organic cities from Section 2.2 to create the evaluation system for Bishan, the table of urban organic composite index evaluation is presented in the following (as shown in Table 4). Finally, the organic composite index and scores of each system and index are calculated (as shown in Table 5).
The organic city of Bishan is mainly indicated by 16 indices of the urban growth boundary, urban scale, urban economy, and urban environment, and they can reflect the overall situation of the city's organic city more comprehensively. By calculating the accumulated variance contribution rate, the scores of 16 indices have different influences on the organic composite index of Bishan, and among them, the natural environment accounts for $10.71 \%$, artificial 
TABLE 3: The explained variance rate of the principal components.

\begin{tabular}{|c|c|c|c|}
\hline \multirow{2}{*}{ Principal component } & \multicolumn{3}{|c|}{ Eigenvalue } \\
\hline & Characteristic root & Variance contribution rate $(\%)$ & Accumulated variance contribution rate (\%) \\
\hline 1 & 30.989 & 68.864 & 68.864 \\
\hline 2 & 6.342 & 14.093 & 82.957 \\
\hline 3 & 2.833 & 6.295 & 89.252 \\
\hline 4 & 1.720 & 3.821 & 93.073 \\
\hline 5 & 1.209 & 2.686 & 95.758 \\
\hline
\end{tabular}

Source: the author.

environment 9.64\%, geology and geography 9.27\%, natural ecology 9.19\%, urban population $7.04 \%$, urban land use $6.53 \%$, human intervention $6.3 \%$, population size $5.09 \%$, population density $4.81 \%$, population structure $4.71 \%$, social environment $4.61 \%$, development intensity $4.33 \%$, main functions $4.13 \%$, industrial structure $3.84 \%$, production process $3.78 \%$, and economic growth $2.90 \%$, respectively.

With the statistics of 16 indices, the statistical results of the actual indices of the urban growth boundary, urban scale, urban economy, and urban environment are presented in Table 6 (as shown in Table 6).

To better compare and analyze the trend of variation and connection between the four systems, different coordinate systems are applied to conduct statistical analysis for the data, which are presented in Figure 3 to highlight the trend of variation of different systems (Figure 4).

4.3. The Analysis of the Urban Organic Composite Index. It can be well observed that the scores for the urban environment increase more rapidly every year, but the growth rate has been slowed in 2017 and 2019. The scores for the urban growth boundary have increased rapidly and even more rapidly from 2013 to 2018. In addition, the scores for the urban scale and economy systems increase more slowly, and these two systems are positively correlated to each other, which indicates that as the urban economy develops, the urban scale is expanding as well.

SPSS is used to conduct PCA on the collected data, and with the variance contribution rate, the corresponding weights for different systems are $38.33 \%$ for the urban growth boundary system, $23.07 \%$ for the urban scale system, $13.64 \%$ for the urban economic system, and $24.96 \%$ for the urban environment system, respectively. Consequently, the urban growth boundary has the greatest influence on the final results of the urban organic composite index, and the urban growth boundary system $>$ the urban environment system $>$ the urban scale system $>$ the urban economic system. The analysis of the urban organic composite index is shown in Figure 5.

With the statistics of the index results of four systems, the scores for the organic composite index of Bishan organic city from 2011 to 2020 can be acquired (as shown in Table 7).

From Figure 6, it can be seen that the average annual growth rate of the organic composite rate of Bishan is $5.31 \%$ for the past 9 years, with the lowest $1.51 \%$ in 2015 and the highest $10.18 \%$ in 2014 .
From the scores of the organic composite index of Bishan, it is obvious that the construction of the organic city in Bishan is rapid, and from 2011 to 2020, the organic composite index scores have even doubled. In addition, from the analysis of the composite index of Bishan, the development of the city relies on the urban environment, reasonable urban growth boundary, and controlled urban scale, which all stimulate the economic development of the city.

\section{Mock Experiments on the Urban Organic City Based on Neural Networks}

The experiments apply the convolutional neural network's residual network ResNet, which is used to predict quickly the organic city of the urban development as shown in Figure 7. Before the deep learning method, the datasets need to be integrated. This research adopts the model-theoretic value simulation methods to generate 3600 datasets in total. In Figure 8, a comparison analysis diagram of data simulation is discussed.

\subsection{The Establishment of the Convolutional Neural Network} Structure. To better address the problem of vanishing or exploding gradients when calculating the backpropagation caused by optimization and at the same to avoid the poor generalization of the network layer, the experiments will apply the convolutional neural network's residual network ResNet.

Firstly, annual data of the indices of the geology and geography, natural ecology, human intervention, population size, urban land use, urban population, development intensity, main functions, production process, artificial environment, population structure, population density, economic growth, industrial structure, natural environment, and social environment are obtained and input.

The data are extracted for features by the network front end backbone for later use. The backbone network adopts ResNet50, with 50 layers of neural networks. It is connected with a pyramid pooling module to extract multiscale information and to fuse the features collected from the overall and partial information. Further, feature data from different levels are merged through skip connection, and finally, with the output network, classification and segmentation are conducted. After comparing the model classification and segmentation results of every training and actual standard 
TABLE 4: Urban organic composite index evaluation table.

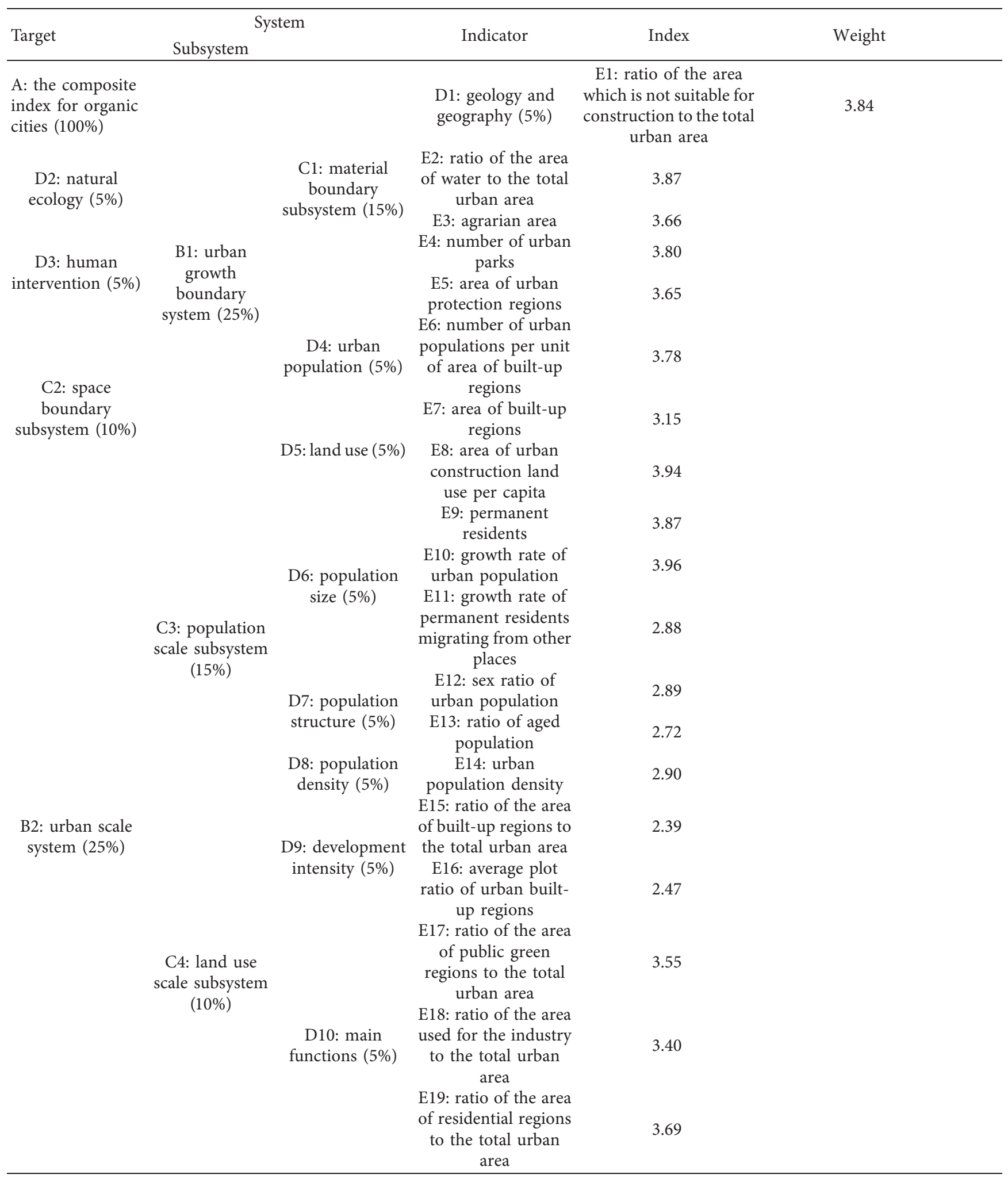


TABle 4: Continued.

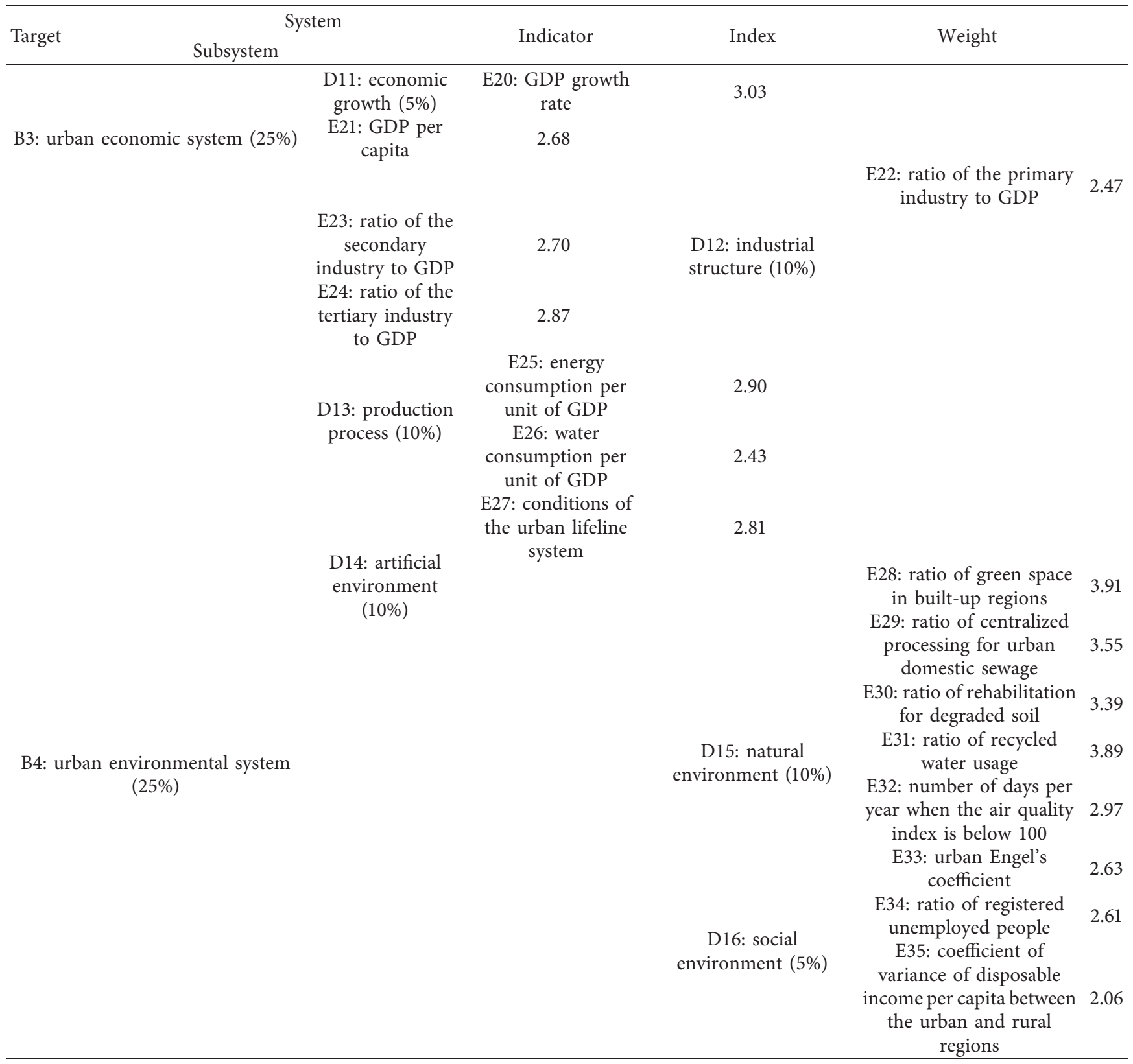

Source: the author.

TABLE 5: The statistical results of Bishan city organic index calculation.

\begin{tabular}{lcccccccccc}
\hline Year & 2011 & 2012 & 2013 & 2014 & 2015 & 2016 & 2017 & 2018 & 2019 & 2020 \\
\hline Geology and geography & 77.31 & 87.33 & 80.86 & 91.93 & 102.34 & 117.79 & 126.55 & 139.51 & 150.46 & 161.9 \\
Natural ecology & 151 & 160.3 & 157.2 & 153.4 & 162 & 161 & 158 & 163 & 167 & 168.9 \\
Human intervention & 189 & 176 & 166 & 163 & 145 & 139 & 135 & 129 & 128 & 125 \\
Urban population & 158 & 166 & 191 & 184 & 177 & 206 & 183 & 192 & 168 & 148 \\
Urban land use & 141.26 & 171.05 & 199.26 & 174.34 & 187.52 & 189.39 & 209.08 & 207.36 & 205.31 & 203.88 \\
Population size & 41.26 & 71.05 & 89.26 & 74.34 & 97.52 & 94.39 & 97.08 & 98.36 & 101.31 & 113.38 \\
Population structure & 85 & 76 & 84 & 92 & 91 & 92.8 & 94 & 94.6 & 96 & 97.3 \\
Population density & 62 & 61 & 63 & 65 & 67 & 68 & 73 & 77 & 79 & 84 \\
Development intensity & 8.9 & 7.63 & 6.66 & 6.33 & 4.53 & 3.91 & 3.35 & 2.99 & 2.38 & 2 \\
Main functions & 2.8 & 2.66 & 1.91 & 1.84 & 1.77 & 2.06 & 1.83 & 1.92 & 1.68 & 1.48 \\
\hline
\end{tabular}


TABle 5: Continued.

\begin{tabular}{lcccccccccc}
\hline Year & 2011 & 2012 & 2013 & 2014 & 2015 & 2016 & 2017 & 2018 & 2019 & 2020 \\
\hline Economic growth & 286.74 & 356.84 & 499.7 & 573.22 & 686.7 & 851.81 & 970.86 & 1220.4 & 1439.49 & 1651.23 \\
Industrial structure & 373.21 & 424.8 & 540.14 & 693.69 & 323.54 & 439.93 & 414.89 & 561.06 & 804.33 & 1010.63 \\
Production process & 220.95 & 261.5 & 313.6 & 350.23 & 365.21 & 425.49 & 466.09 & 526.52 & 601.61 & 675.01 \\
Artificial environment & 386.74 & 456.84 & 599.07 & 673.22 & 786.7 & 951.81 & 1070.86 & 1322.4 & 1539.94 & 1751.23 \\
Natural environment & 378.13 & 450.66 & 690.78 & 779.16 & 923.55 & 1126.21 & 1252.1 & 1493.1 & 1832.23 & 2026.81 \\
Social environment & 330.59 & 371.6 & 403.06 & 460.23 & 476.33 & 535.95 & 587.19 & 636.25 & 702.16 & 786.11 \\
\hline
\end{tabular}

Source: the author.

TABLE 6: The statistical results of Bishan city organic index calculation.

\begin{tabular}{|c|c|c|c|c|c|c|c|c|c|c|}
\hline Year & 2011 & 2012 & 2013 & 2014 & 2015 & 2016 & 2017 & 2018 & 2019 & 2020 \\
\hline Urban growth boundary system & 77.31 & 101.33 & 80.86 & 101.93 & 122.34 & 147.79 & 186.55 & 229.51 & 240.46 & 301.9 \\
\hline Urban scale system & 15 & 16 & 22 & 21 & 23 & 28 & 35 & 41 & 48 & 52 \\
\hline Urban economy system & 33.6 & 37.1 & 40.3 & 46.2 & 47.6 & 53.5 & 58.7 & 63.6 & 70.2 & 78.6 \\
\hline Urban environment system & 231.48 & 271.06 & 304.6 & 305.2 & 307.33 & 403.9 & 408.7 & 503.2 & 602.1 & 608.3 \\
\hline
\end{tabular}

Source: the author.

Geology and geography

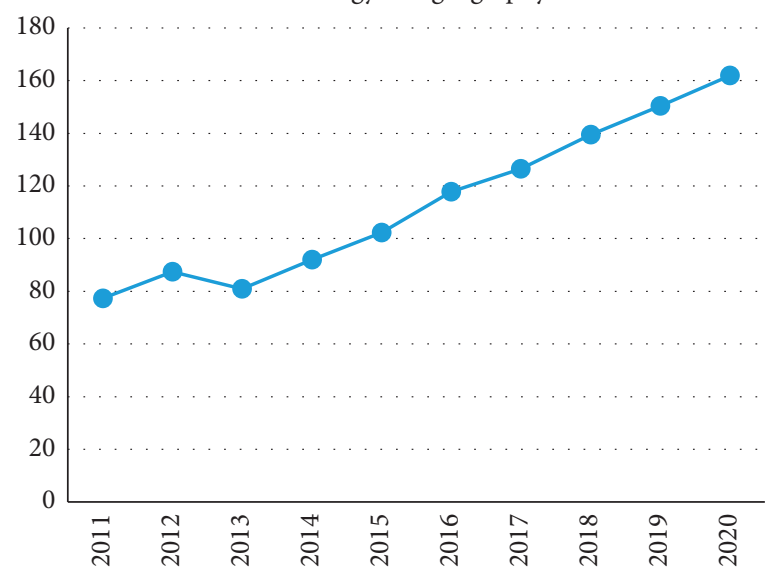

Human intervention

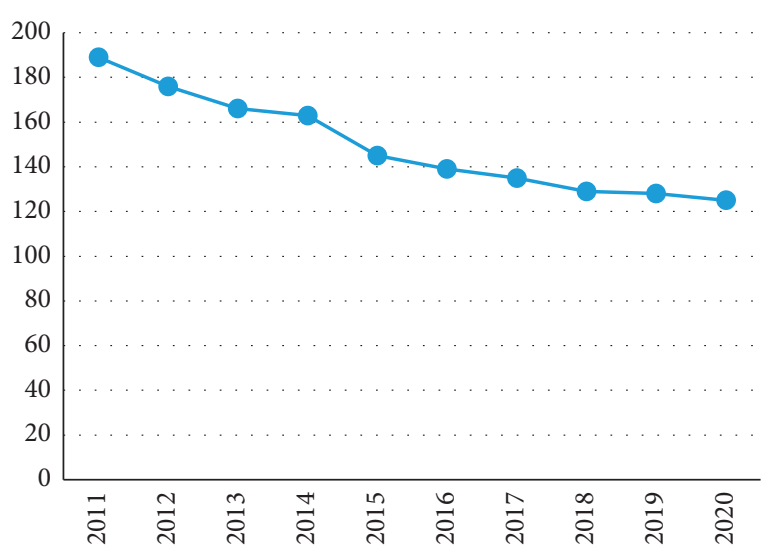

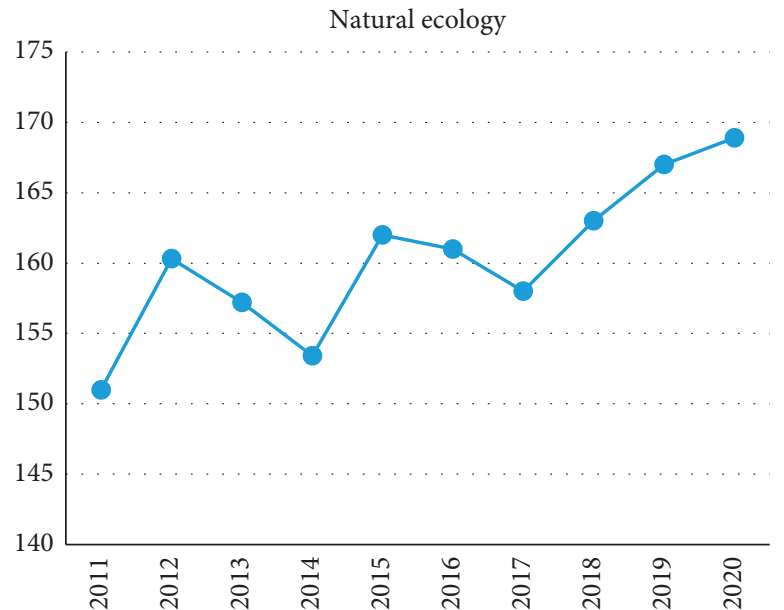

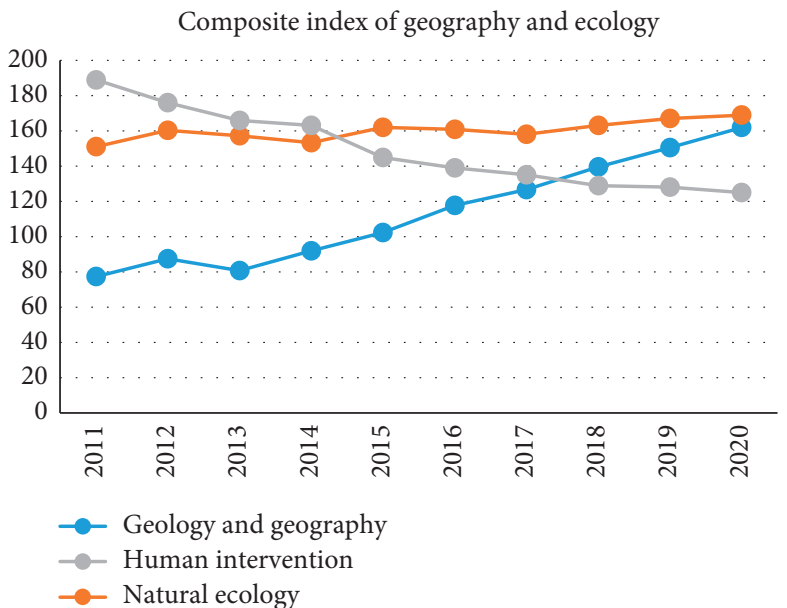

FIGURE 3: The CNN analysis results of geographic and ecological indices. Source: the author.

data, the loss value of the loss function is minimized. The weighted values of nodes in the neural network are consistently adjusted to make the prediction closer to the real model. A well-trained neural network can be used to predict quickly the organic city of the urban development (as shown in Figure 7).

The urban organic index datasets are input into the network front end backbone, and according to the 

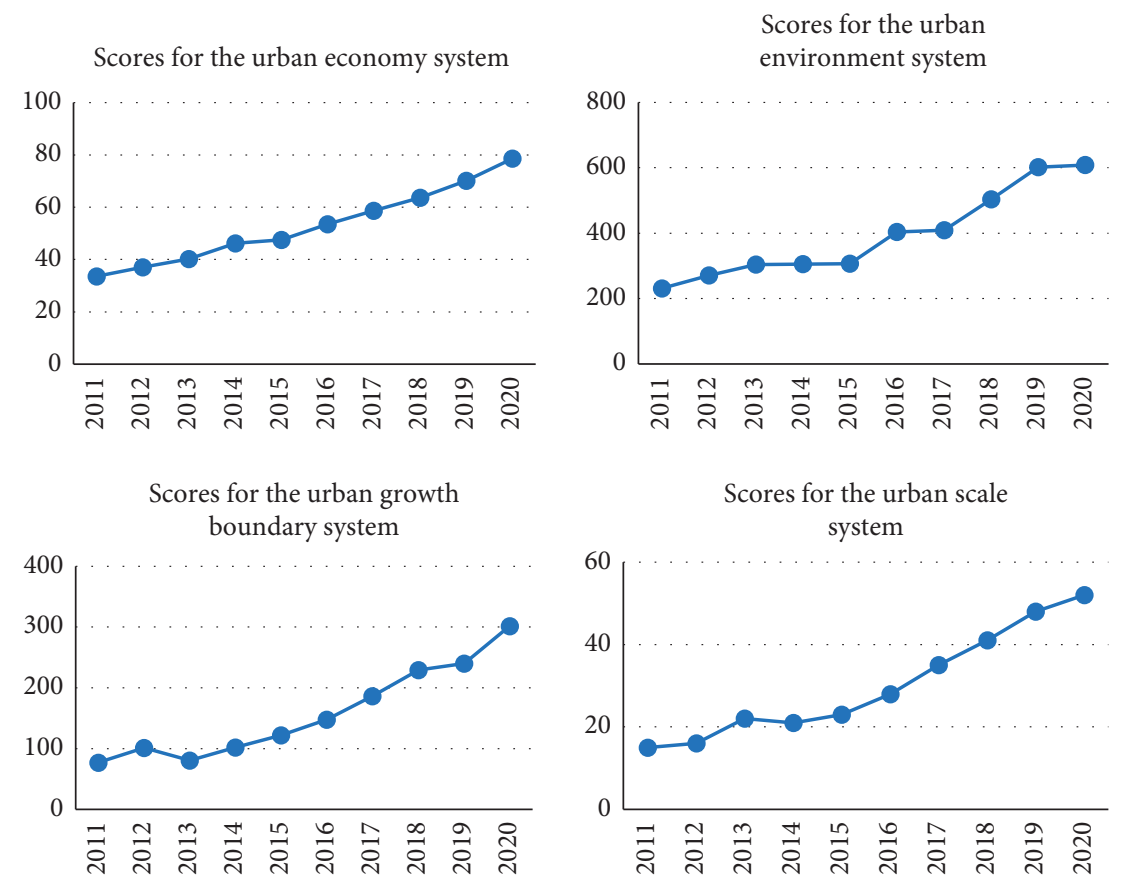

FIGURE 4: The scores of the urban organic composite index system in Bishan. Source: the author.

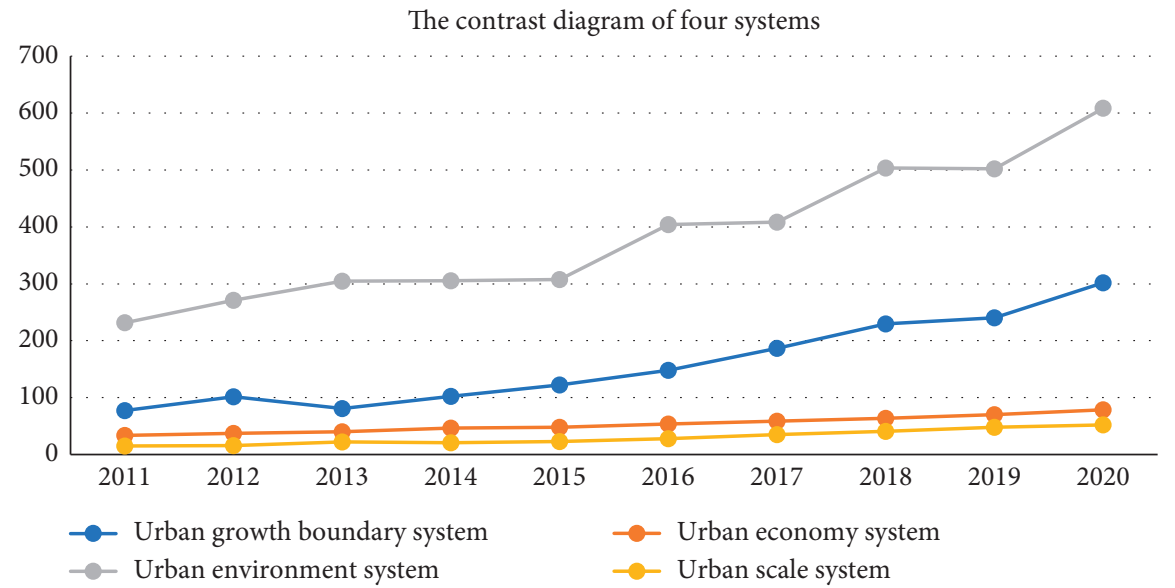

Figure 5: The contrast diagram of four systems. Source: the author.

TABLE 7: The urban organic composite index evaluation scores for the past 10 years.

\begin{tabular}{|c|c|c|c|c|c|c|c|c|c|c|}
\hline Year & 2011 & 2012 & 2013 & 2014 & 2015 & 2016 & 2017 & 2018 & 2019 & 2020 \\
\hline An organic composite index score & 289.3475 & 306.3725 & 311.94 & 418.5825 & 425.0675 & 458.2975 & 472.2375 & 509.3275 & 540.19 & 560.2 \\
\hline
\end{tabular}




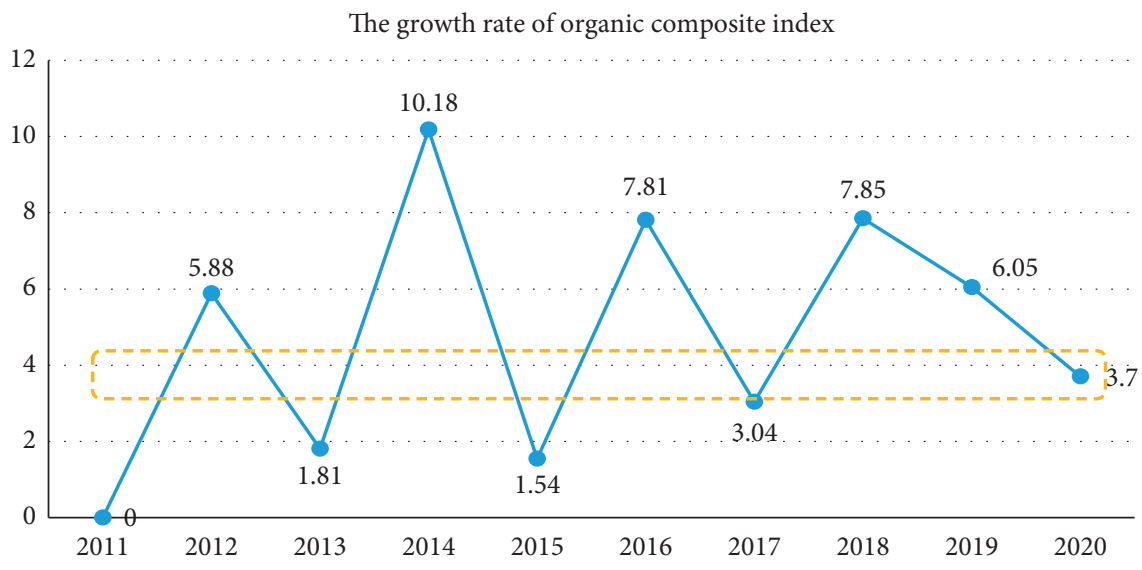

FIgURE 6: Bishan comprehensive index of organic growth rate. Source: the author.
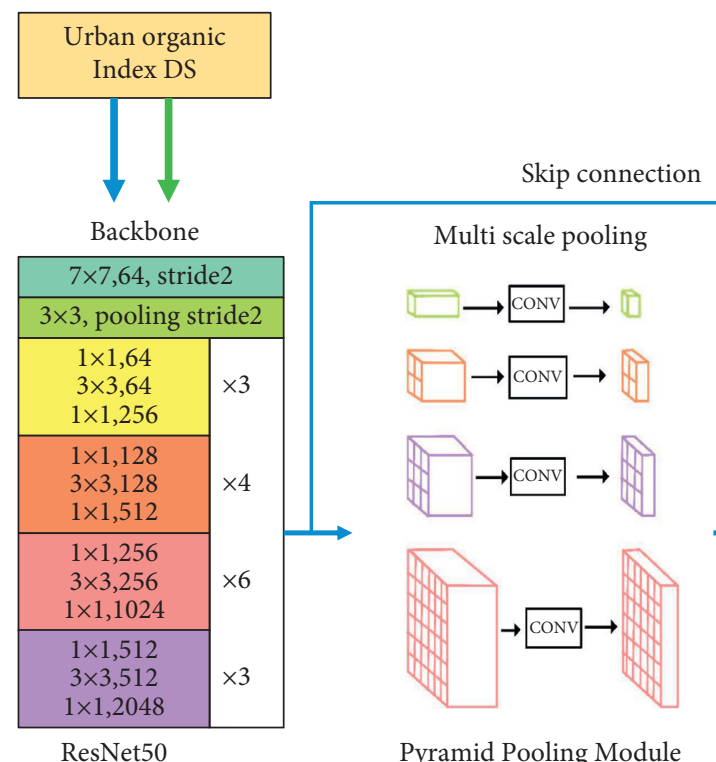

Pyramid Pooling Module
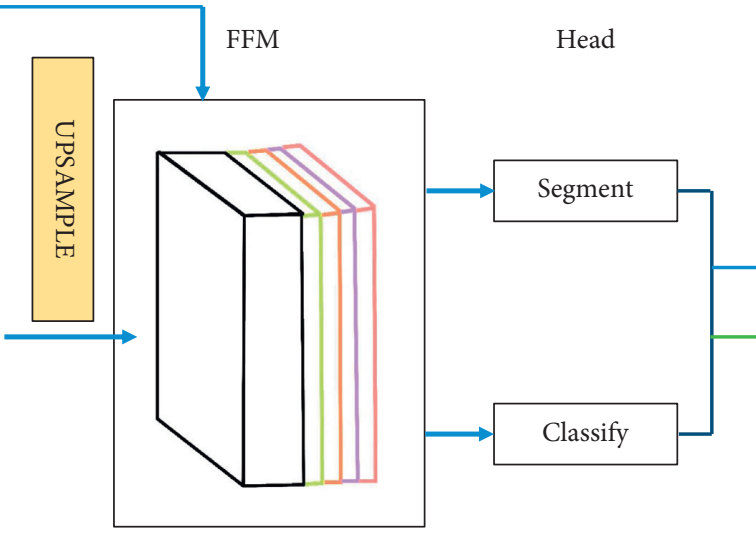

CONCAT

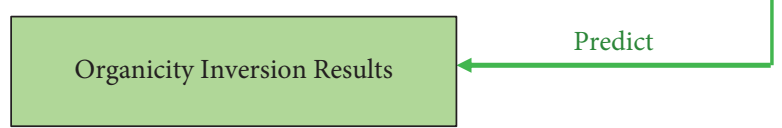

FIGURE 7: Neural network structure diagram of urban organic index DS retrieval method. Source: the author.
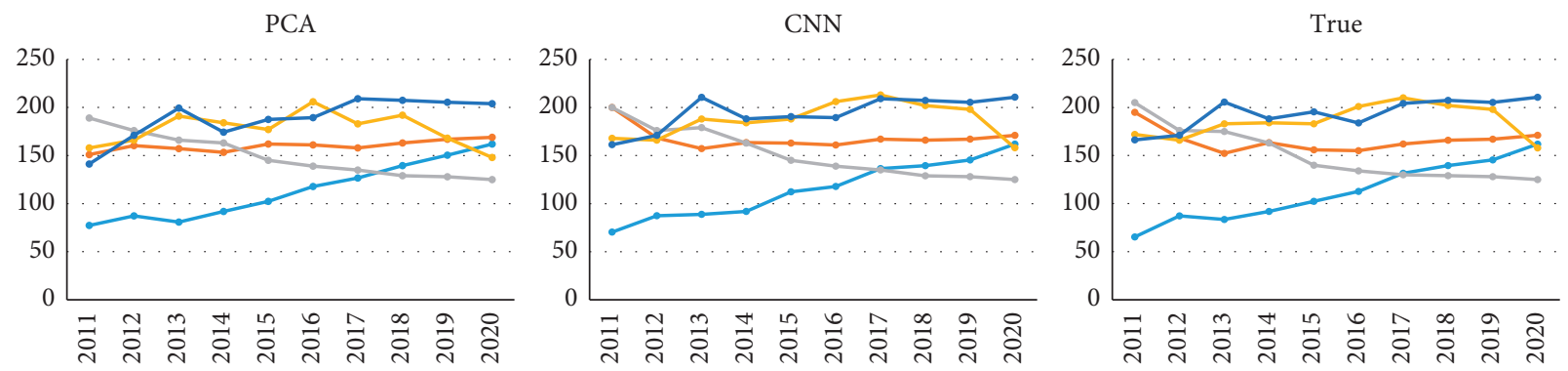

Figure 8: Comparison analysis diagram of data simulation. Source: the author. 
backpropagation formula, when the value of the input layer is extremely great, the gradient value of the input layer calculated by the backpropagation will become extremely great, causing a lower learning efficiency and large consumption of time. So, when establishing the convolutional neural network (CNN) model, the experiments will firstly batch normalize $(\mathrm{BN})$ the data information, the data processing for hidden layers will perform the BN processing as well, and after the BN processing, the network will have the most optimized computational process.

The $\mathrm{BN}$ algorithm is shown as follows:

Batch processing input $x$ (min-batch):

$$
\boldsymbol{\beta}=\left\{\mathbf{x}_{1}, \mathbf{x}_{2}, \ldots, \mathbf{x}_{\mathbf{n}}\right\} \text {. }
$$

Standard network input:

$$
\mathbf{y}_{\mathrm{i}}=\left\{\mathrm{BN}_{\mathrm{y}}, \boldsymbol{\beta}\left(\mathrm{x}_{\mathrm{i}}\right)\right\} \text {. }
$$

(1) Calculate the mean of the batch processing data:

$$
\mu_{\rho} \longleftarrow \frac{1}{\mathbf{m}} \sum_{\mathbf{i}=1}^{\mathrm{m}} \mathbf{x}_{\mathrm{i}}
$$

(2) Calculate the variance of the batch processing data:

$$
\boldsymbol{\sigma}_{\boldsymbol{\beta}}^{2} \longleftarrow \frac{1}{\mathbf{m}} \sum_{\mathbf{i}=1}^{\mathbf{m}}\left(\mathbf{x}_{\mathbf{i}}-\boldsymbol{\mu}_{\beta}\right)^{2} .
$$

(3) Standardization:

$$
\mathbf{x}_{\mathbf{i}} \longleftarrow \frac{\mathbf{x}_{\mathbf{i}}-\mu_{\beta}}{\sqrt{\sigma_{\beta}^{2}+\varepsilon}} .
$$

(4) The variation and deviation of the scale:

$$
\mathrm{y}_{\mathrm{i}} \longleftarrow \gamma \mathbf{x}_{\mathrm{i}}+\boldsymbol{\beta}=\mathrm{BN}_{\gamma}, \boldsymbol{\beta}\left(\mathrm{x}_{\mathrm{i}}\right)
$$

(5) Returned values: the scale factor $\gamma$ and shift factor $\beta$ in the learning parameters.

The data information of three channels passes the network front end backbone module ResNet50 and is filtered 64 times by a $7 * 7$ convolutional filter to obtain data of 64 channels. Further, the data are pooled by a $3 * 3$ pooling window and go through convolutional computation 48 times, the number of channels is expanded to 2048, and these steps can maximize the ability to extract the features from the data information. The data are then calculated in the pyramid pooling module after forward-propagation and pooled by $1 * 1,2 * 2,3 * 3$, and $6 * 6$ pooling windows, respectively, to acquire multiscale data feature maps. Furthermore, the number of channels is reduced after the convolutional computation of a $1 * 1$ filter, and the output data are sampled by bilinear interpolation and enter the module of feature fusion, where either the channels will be concatenated (Concat for short) or the corresponding feature maps are added for convolutional operation. Suppose the input channels are $\mathbf{x}_{1}, \mathbf{x}_{2}, \ldots, \mathbf{x}_{\mathbf{n}}$ and $\mathbf{y}_{1}, \mathbf{y}_{2}, \ldots, \mathbf{y}_{\mathbf{n}}$, Concat processing is defined as a convolutional computation on the input.

$$
\text { Concat }=\sum_{\mathbf{i}}^{\mathbf{n}} \mathbf{x}_{\mathbf{i}} * \mathbf{k}_{\mathbf{i}}+\sum_{\mathbf{i}}^{\mathbf{n}} \mathbf{y}_{\mathbf{i}} * \mathbf{k}_{\mathbf{i}+\mathbf{n}} \text {. }
$$

When the data enter the output end through backpropagation, the data need semantic segmentation to divide the data results into possible classifications. As for the binary classification, the last layer of the neural network adopts the Sigmoid activation function and the Softmax function for multiple classifications. This research applies the Softmax activation function. During the classification, the function maps the output of various neurons to the range $(0,1)$ to complete the classification. Taking into account the training duration and difficulty, the research aims to normalize the output end of the data to a discretized classification, and different from the continuous output values of regression, the classification's neural network output is a series of discrete values because the input data are a series of discrete frequency data. Softmax is more suitable for the training and prediction of discrete values, and at the same time, the mean square error (L2 norms) loss function that is commonly used in regression is added to the target function.

The Softmax function is defined as follows:

$$
\mathbf{a}_{j}=\frac{\mathrm{e}^{\mathrm{zj}}}{\sum_{\mathrm{i}} \mathrm{e}^{\mathrm{zj}}}
$$

where $\mathbf{z}_{\mathbf{j}}$ stands for the input of $j$ neurons in the last network layer, $\mathbf{a}_{\mathbf{j}}$ stands for the output of $j$ neurons in the last layer, the natural number $e$ is used to intensify the difference of the possibilities, and $\sum_{\mathbf{i}} \mathrm{e}^{\mathbf{z j}}$ is the sum of all neurons in the last layer. The Softmax function will judge the output of the neurons in the last layers with the possibility distribution, and the higher the possibility of a certain neuron is, the better authenticity the classification that the neuron corresponds to has.

5.2. Training and Prediction. Before the deep learning method, the datasets need to be integrated, and for the field of computer vision, the dataset training is open and accurately calibrated and calls for millions of datasets. However, in real life, relevant data of the urban organic composite index, either measured on the spot or converted, are limited. As a consequence, this research adopts the model-theoretic value simulation methods to generate 3600 datasets in total.

The datasets are classified into 16 types according to the combination of urban organic composite indices, and they are geology and geography, natural ecology, human intervention, population size, urban land use, urban population, development intensity, main functions, production process, artificial environment, population structure, population density, economic growth, industrial structure, natural environment, and social environment. These 16 classifications correspond to the annual combined data (of 10 years), and finally, 160 datasets are designed.

(i) First, after determining the content of the model, 160 times numerical simulations are performed, which is realized by the codes through stochastic model repeated computation. 
(ii) Second, the data of organic parameters are sorted into 160 pairs of models, among which the datasets are the input samples, the true model is the calibrated datasets, and the corresponding relationship between the input datasets and calibrated datasets is guaranteed.

(iii) Finally, the trained datasets are input into the designed convolutional neural network for repetitive training to perfect the weight distribution of each node in the convolutional neural network. After several rounds of training, the data which await inversion is input into the neural network to realize the data prediction and the inversion result.

5.3. Numerical Simulation. Five datasets are chosen randomly from the research model to serve as the example of the convolutional neural network inversion, and the results are presented in Figure 8 (as shown in Figure 8), where the first chart is the urban organic composite index (results of PCA), the second is the inversion results of deep learning, and the third is the true data.

It can be well observed that the measurement and prediction of both PCA and CNN are close to those of the true data, and the prediction of $\mathrm{CNN}$ is better than that of PCA. PCA conducts data computation in terms of the weights, while CNN does according to the original database, and hence $\mathrm{CNN}$ has better accuracy. From the figure, the indices for the human intervention (blue) and urban population (yellow) have the greatest fluctuation and variance. By comparing the original input data of these two pairs of data, PCA has a worse accuracy for factors associated with humans but a better accuracy for factors that involve less human influence. So, we can confirm that the deep learning convolutional neural network can accurately realize nonlinear inversion for the organic city data of small cities in China, and as for conditions with more complex data and various human interventions, it can still achieve rather a good inversion.

\section{Analysis of the CNN Experiment Results}

6.1. Analysis of the Influence of the Urban Geographical and Geological Structure. After comparing the indices of the geology and geography, natural ecology, and human intervention in Bishan, we find that, from 2011 to 2020 (as shown in Figure 3), the geology and geography index has increased steadily every year, and the urban construction of the city has set more store by the original geological and geographical conditions. In addition, the natural ecology index with less variation indicates that the original natural ecological environment has been protected during the urban construction and the impact of urban construction on the natural environment has been minimized. Further, the annually decreasing human intervention index implies that the urban construction of Bishan has adapted to the local conditions, integrating the development of both the city and nature.
6.2. Analysis of the Urban Industrial and Economic Structure. Comparing the indices of the economic growth, industrial structure, and production process (as shown in Figure 9) from 2011 to 2020, the economic growth index has increased more rapidly every year, indicating that Bishan's economy remains in a phase of accelerating development. The production process index has shown an accelerating trend from 2017 based on a steady increase because the production process has been upgraded since the adjustment of Bishan's industrial structure. However, the industrial structure index has gone through several phases of fluctuation and reached temporary peaks in both 2014 and 2020 and hit rock bottom in 2015, which indicates that the two phases of Bishan's industrial development have occurred before 2014 and after 2017.

6.3. Analysis of Urban Population Development Structure. In terms of the population, the urbanization rate of Bishan at the end of 2012 was $32.8 \%$, and since then, Bishan has entered the accelerating phase of urban development. From Figure 10 (as shown in Figure 10) from 2014 to 2017, the indices of the urban population size, structure and density, and land use have simultaneously increased rapidly, indicating that the urbanization process of Bishan has stimulated the development speed of the urban land and the expansion of the urban scale. After 2018, the land use index has started to drop, while the urban population index has kept increasing, implying that the large-scale urban construction has been slowed, but the population bonus brought by the urbanization still exists, and therefore with the continuous population urbanization, the quality of it has been improved as well. Further, due to the combination of urban development, geology and geography, and the natural ecological environment, the rapid expansion of the city is controlled, and hence the population urbanization does not lag behind the land urbanization, and the city develops in an orderly manner.

\section{Comparison Analysis}

To guarantee that the results of the research enjoy some generality for the organic city evaluation of small cities in China and verify the stability of the data computation of urban organic index in the CNN model, this paper conducts a data measurement analysis for Jiangyin (small city), Jiangsu Province, at the same time.

This analysis, similarly, first adopts PCA to measure and calculate the urban organic composite index and then input the datasets formed by the organic index into the established CNN model.

7.1. Influence of the Urban Geographical Environment on the Construction of Organic Cities. After comparing the indices of the geology and geography, natural ecology, and human intervention between Bishan and Jiangyin output by CNN computation, we find that when the original geological and geographical conditions of a city are complex, the natural ecological environment is good, the urban development is limited by the geological and 

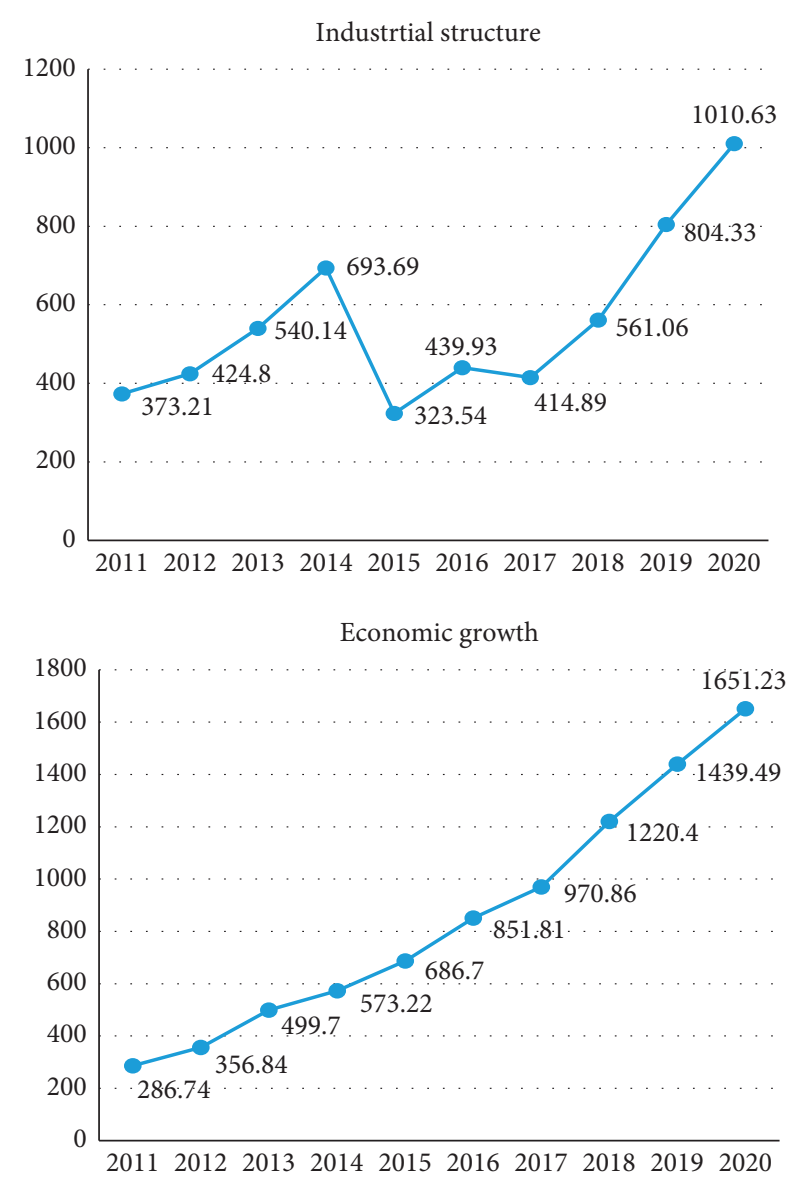
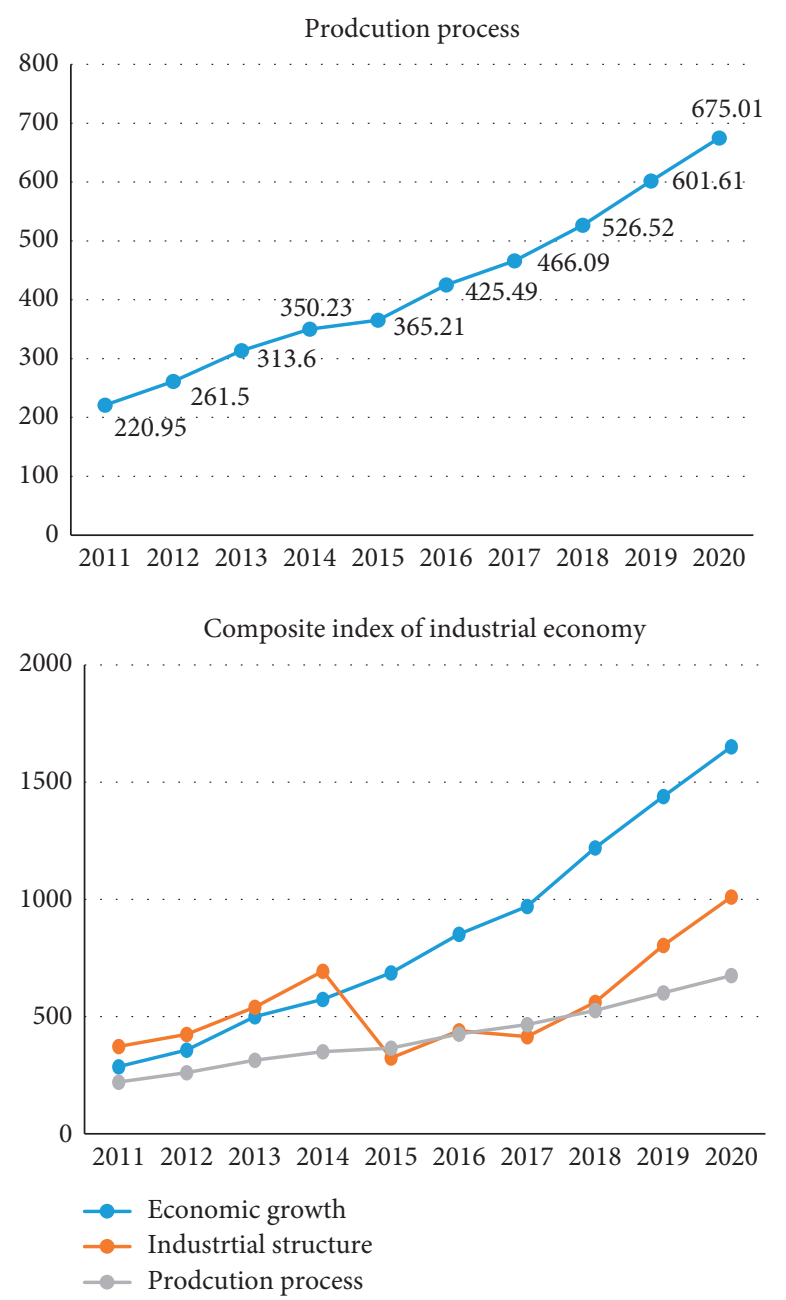

FIgURE 9: CNN analysis result pictures of industrial economy. Source: the author.

geographical environment, the urban construction which is featured with human intervention is scarce, and the urban development tallies better with the geographical and natural ecological environment. When the original geological and geographical conditions are simple, the city has some natural ecological resources, the urban development needs to specify different urban growth boundaries for different phases of urban development, and the urban development needs to take into account both the protection and maintenance of the natural ecological environment, causing some human intervention, but the intervention will gradually decrease with the urban development and protection of the nature (as shown in Figures 11 and 12).

In conclusion, the geographical and natural environment not only lays the foundation for the construction of organic cities but also determines the basic form and path for the construction of organic cities.

7.2. The Influence of the Urban Economic and Industrial Structure on the Construction of Organic Cities. Comparing the indices of the economic growth, industrial structure, and production structure between Bishan and
Jiangyin, though the economic growth index of Bishan is greater than that of Jiangyin, its other two indices are both smaller than those of Jiangyin. It can be inferred that the economic growth cannot sufficiently reflect the conditions of the urban industrial structure and production process. The adjustment and upgrading of the industrial structure can directly lead to the upgrading and transformation of the production process. Further, in Bishan with the original economic basis and industrial structure, the ecology and industrial structure have been improved to boost the rapid economic growth, while the ratio of the primary, secondary, and tertiary industry to the entire industrial structure needs to be optimized. By contrast, in Jiangyin, the organic sustainable industry system is brought up based on the original industrial structure, and the technological upgrade of the secondary industry improves the production efficiency. The ratio of the tertiary industry increases to augment the level of the whole urban industrial structure, leading to a benign development and sustainable growth of the urban economy (as shown in Figures 13-15).

Through the comparison of these two cities, we find that different economic bases will have different effects as the urban organic economy develops. When the city is in a period of economic growth due to the adjustment of the economic 

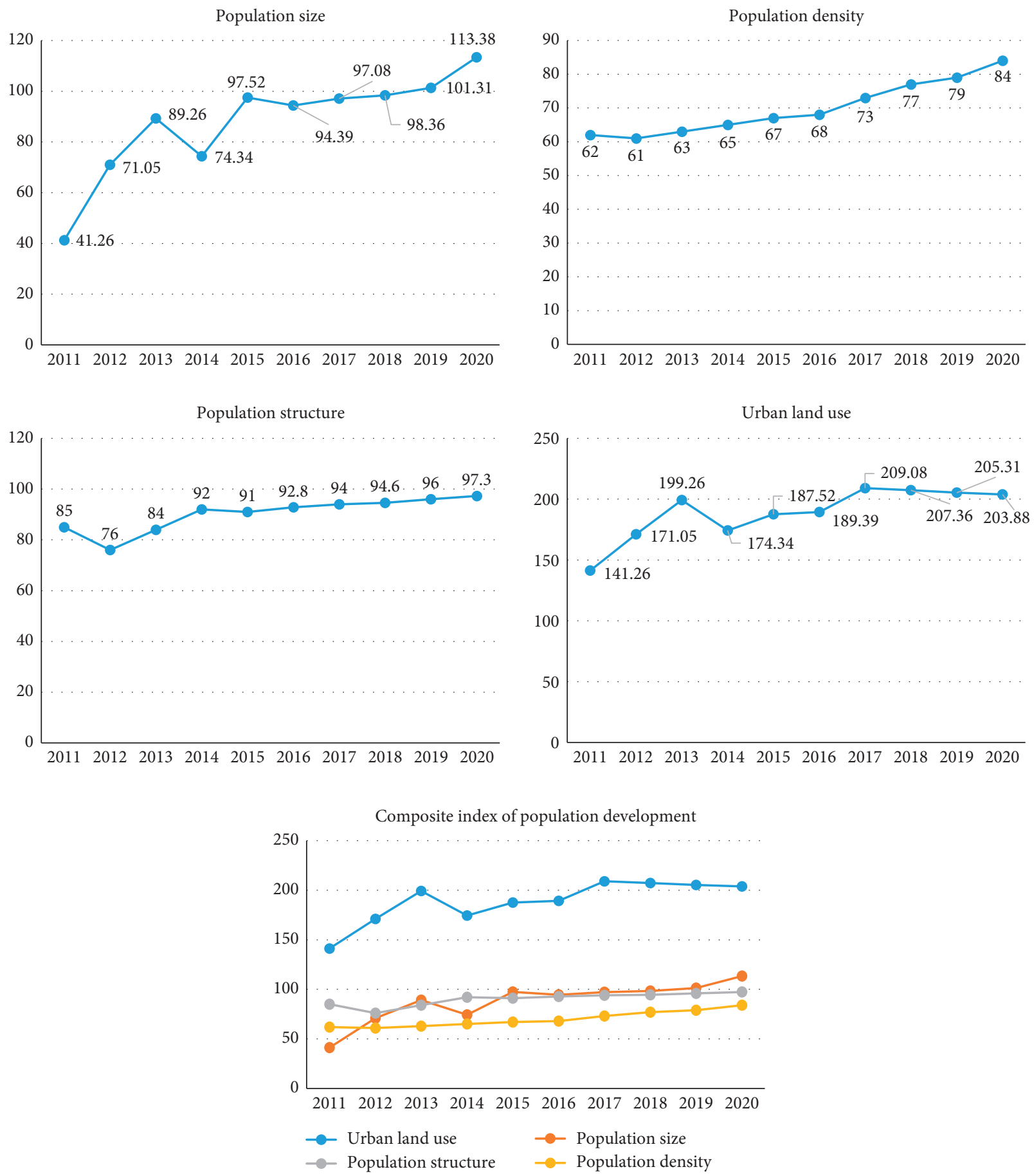

FIgURE 10: CNN analysis results of population development. Source: the author.

industrial structure, the adjustment will boost the rapid growth of the economy. However, when the city is in the period of maturity, the upgrading and transformation of the industrial structure and the innovation of the production process and technology are both needed to stimulate the city's economy. In summary, though the urban economy and industrial structure are the economic foundation for the construction of organic cities, the modes of the economic development and industrial structure should be consistent with the development period of the city.
7.3. The Influence of the Urban Population on the Construction of Organic Cities. In terms of population, the urbanization rate of Bishan was $32.8 \%$ at the end of 2012, and since then, Bishan has entered the period of accelerating development. According to the statistics of index scores presented in Figure 16, from 2014 to 2017, the four indices of Bishan show a trend of fast growth simultaneously, indicating that the promotion of the urbanization process in Bishan has spurred the development and construction speed of the urban land use and also the expansion of the urban scale. After 2018, 


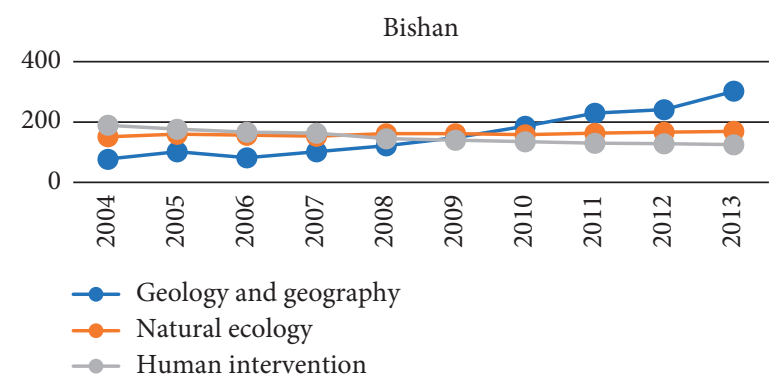

FIGURE 11: Comprehensive analysis of geological and geographical index, natural ecological index, and human intervention index in Bishan. Source: the author.

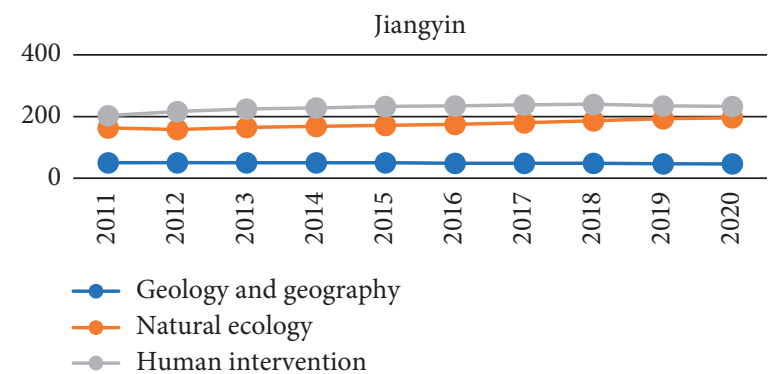

Figure 12: Comprehensive analysis of geological and geographical index, natural ecological index, and human intervention index in Jiangyin. Source: the author.

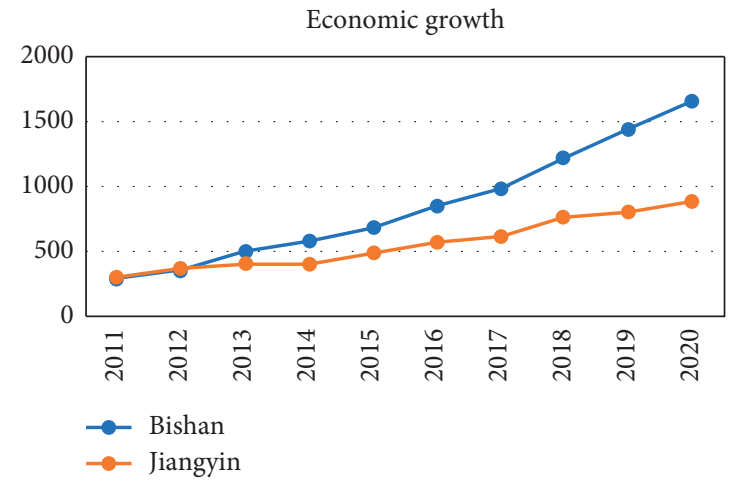

FIgURE 13: Comparison of the economic growth index. Source: the author.

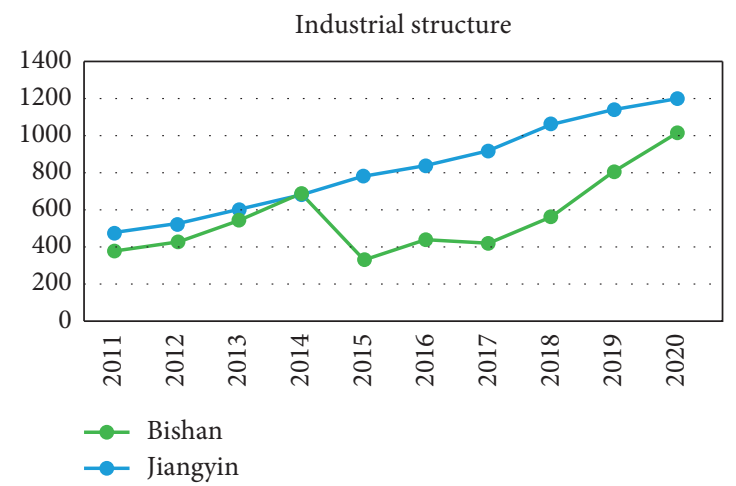

Figure 14: Comparison of the industrial structure index. Source: the author. 


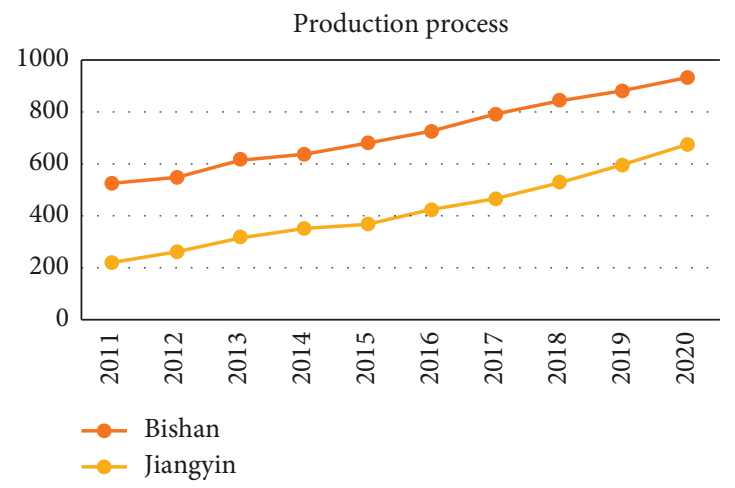

FIgURE 15: Comparison of the production process index. Source: the author.

however, the land use index has started to drop every year, while the indices of the urban population have kept increasing. It demonstrates that the large-scale urban construction in Bishan has been slowed since 2018, but the population bonus due to the population urbanization still exists, leading to an improved urbanization quality with the continuous population urbanization. In addition, because the urban development of Bishan goes well with the geological and geographical conditions and natural ecological environment, the fast expansion of the city is controlled, and hence the population urbanization does not lag behind the land urbanization, and the city is developing in an orderly manner.

From Figure 17, it can be seen that the relationship between the urban population and land use in Jiangyin can be classified into 2 major phases, before 2017 and after 2017, respectively. Before 2017, the growth of the urban population size spiked in 2013 and touched bottom in 2017, while the scores of the urban population density and structure increase annually and positively correlated with the scores of the urban land use index. Hence, it can be inferred that the population of Jiangyin has reached a certain size and the urban scale is less influenced by the population size, but the urban population density and structure will lead to new functional requirements for the city. Hence, the index of the urban land use is boosted. After 2017, the urban population size has started again to increase and the other three indices have shown a general increasing trend as well. Since 2017, due to a new round of planning and implementation of overall rules, the urban function of Jiangyin has been further improved, the optimization of the urban industrial structure has again promoted population urbanization, and the demand for land has become outstanding. In summary, for Jiangyin, which is about to enter the mature period of urban development, the changes of the urban scale are mainly influenced by urban functions, but if the development of the urban scale is not well controlled, the population urbanization will lag behind the land urbanization, and hence the speed of the urban development and construction is the key for the construction of organic cities for Jiangyin.

Considering both the cases of Bishan and Jiangyin, the scales of urban population and land use will have different influences on the development of the city in different phases of urban development. When the city just enters the period of fast development, the scale of the urban population has a greater influence, and the city stays in a period of fast growth. However, when the city approaches or enters the phase of mature development, the scale of urban land use will have a greater influence, and the city enters a phase of internal optimization. In short, the urban population's influence on the construction of organic cities mainly takes place in the period of fast urban development; a reasonable rate of growth of urban population can effectively control the growth of urban size for urban construction to proceed in an orderly manner.

\subsection{The Influence of the Urban Development Structure on the} Construction of Organic Cities. The urban growth boundary system of organic cities consists of the indices of geology and geography, natural ecology, human intervention, urban population, and urban land use.

From the pie charts (as shown in Figure 18), the urban growth boundary has a major influence on the urban development of Bishan, mainly due to the geological and geographical conditions and the natural ecological environment of the surrounding area of the city. Jiangyin's urban organic development is less influenced by the urban growth boundary, mainly because the city is located in the plain with simple geological and geographical conditions, and the urban growth boundary is principally specified artificially (as shown in Figure 19).

The urban scale system of organic cities consists of the indices of the population size, population structure, population density, development intensity, and main functions (as shown in Figure 20).

Through the comparison, Bishan is the period of fast development, and the urban population size has a comparatively greater influence on the urban scale, while in Jiangyin, which is in the period of maturity, the urban population has gained a certain scale, and therefore, the city's development is mainly focused on the urban land use and the influence of the urban main functions on the urban scale serves well as the proof (as shown in Figure 21). 


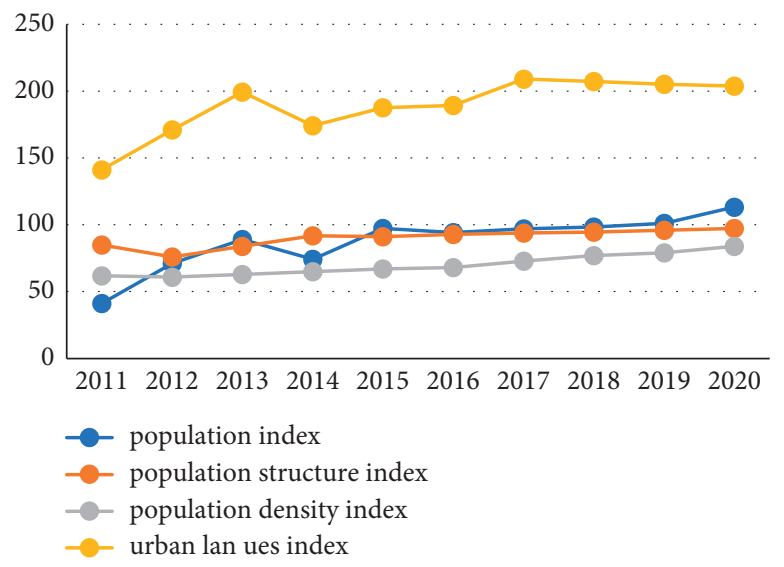

FIgURe 16: Population index, population structure index, population density index, and urban land use index in Bishan. Source: the author.

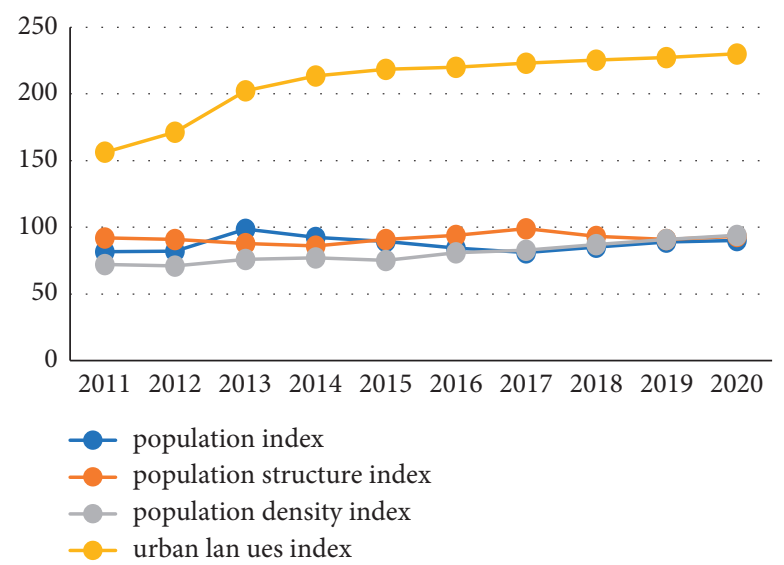

Figure 17: Population index, population structure index, population density index, and urban land use index in Jiangyin. Source: the author.

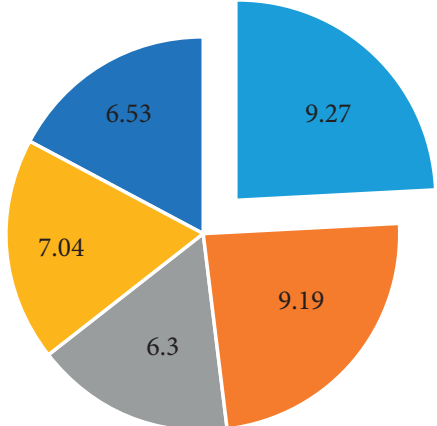

Geology and geography

- The natural ecology

- Human intervention

The urban population

- Urban land

FIGURE 18: Composition of the urban growth boundary in Bishan. Source: the author. 


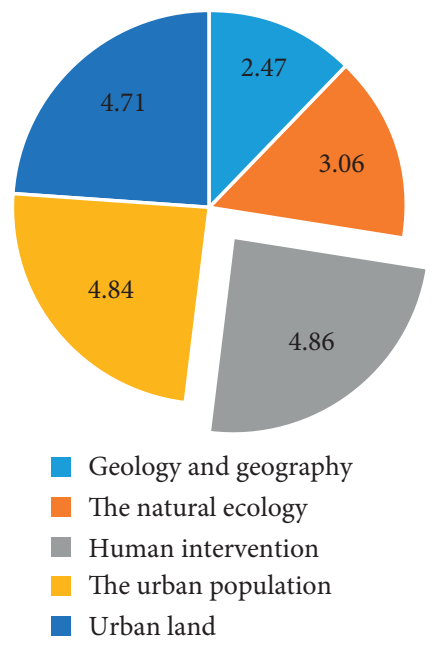

FIgURE 19: Composition of the urban growth boundary in Jiangyin. Source: the author.

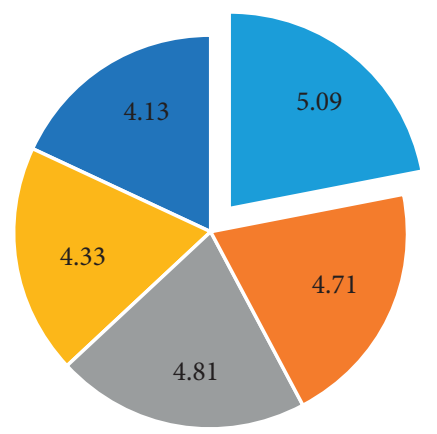

- Population

- The Population structure

The Population density

The intensity of the development

- The main function

Figure 20: Composition of the urban scale system in Bishan. Source: the author.

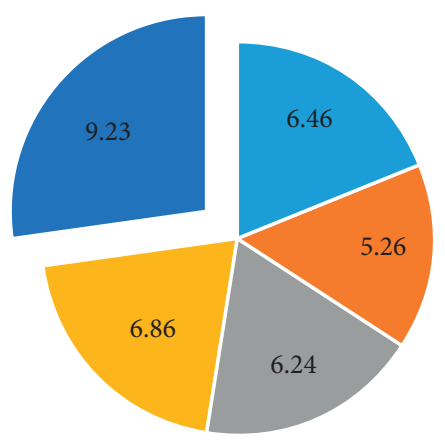

- Population

The Population structure

The Population density

The intensity of the development

- The main function

Figure 21: Composition of the urban scale system in Jiangyin. Source: the author. 


\section{Conclusions}

This research applies the principal component analysis (PCA) to create an evaluation index system for organic cities and improves the evaluation methods for the urban organic city. In addition, the research comes up with a method combining PCA and the convolutional neural network's residual network (ResNet) to establish an optimized model for calculating urban organic cities and chooses Bishan and Jiangyin for the case study.

The main conclusions are as follows:

(1) It is necessary to establish an evaluation index system for organic cities to describe and reflect the organic city and present development of a city and also to monitor and assess the planned development of a city. This research establishes an evaluation system, which can make an objective evaluation of the existence of an organic city for urban construction.

(2) In the process of urban construction, there exist rigid conditions which can be quantified but also flexible ones that cannot be fully quantified. The research, when establishing the evaluation index system, absorbs PCA to avoid subjectivity in the evaluation process and therefore improves the scientificity and accuracy of the evaluation results.

(3) The research methodology also absorbs ResNet, and the network strengthens the functions of the network's convolutional module, and as the network layer deepens, it introduces the residual connection to effectively address the problem of the training's increasing errors caused by too many layers in the real network training and ultimately improves the accuracy of the prediction for the organic cities.

(4) In the research, we choose 2 small cities in China to conduct a case study, and the results prove the efficacy of the method in evaluating the organic city of a small Chinese city.

\section{Data Availability}

The data used to support the findings of this study are included within the article.

\section{Conflicts of Interest}

The authors declare that they have no conflicts of interest.

\section{Acknowledgments}

This study was supported by to the Research on the Conservation of Anti-War Cultural Sites in Beibei District, Chongqing Based on Building Information Modeling Technology Foundation (no. 21SKGH297).

\section{References}

[1] C. Guo, Z. Peng, and J. Ding, "Construction of DEA indicators for comprehensive evaluation of sustainable development," China Population-Resources and Environment, vol. 26, 2016.
[2] X. Sun, X. Liu, F. Li, and Y. Tao, "Comprehensive evaluation of sustainable development of cities of different scales in China," Ecological Journal, vol. 77, pp. 56-59, 2015.

[3] R. Chen, Z. Zhao, D. Xu, and C. Yi, "Progress of research on sustainable development index of cities and urban agglomerations," Advances in Geosciences, vol. 32, pp. 88-92, 2021.

[4] H. Li and L. Yu, "Study on the construction of eco-city evaluation index system in China," Urban Development Research, vol. 63, pp. 81-86, 2011.

[5] Organization for Economic Co-Operation and Development (OECD), Environmental Indicators: A Preliminary Set, OECD, Paris, France, 1991.

[6] United Nations, Indicators of Sustainable Development: Guidelines and Methodologies, United Nations, New York, NY, USA, 3rd edition, 2007.

[7] Y. Huang, X. Zhang, S. Cui, W. Huang, and X. Su, "Construction and weighting of green city evaluation index system," Journal of Environmental Science, vol. 121, pp. 91-94, 2020.

[8] P. Dou, S. Zuo, Y. Ren, and W. Huang, "Construction of green city index system based on city classification," Journal of Ecology, vol. 129, pp. 42-45, 2019.

[9] J. Li, Study on the Model Selection and Comprehensive Benefit Evaluation of Urban Renewal: An Example of Urban Village Transformation in Guangzhou, South China University of Technology, Guangzhou, China, 2019.

[10] P. Wang, Study on the Comprehensive Evaluation and Planning Path for the Renewal of Old Urban Residential Areas, Southeast University, Nanjing, China, 2019.

[11] G. K. L. Lee and E. H. W. Chan, "Factors affecting urban renewal in high-density city: case study of Hong Kong," Journal of Urban Planning and Development, vol. 134, no. 3, pp. 140-148, 2008.

[12] C. K. Cheung and K. K. Leung, "Retrospective and prospective evaluations of environmental quality under urban renewal as determinants of residents' subjective quality of life," Social Indicators Research, vol. 85, no. 2, pp. 223-241, 2008.

[13] D. Liu, "Evaluation of urban environmental sustainability based on two-level fuzzy integrated evaluation," Statistics and Decision Making, vol. 107, no. 18, pp. 56-59, 2014.

[14] B. Ning, W. Peng, and S. Guo, "Comprehensive evaluation of environmental sustainability in western mining cities based on entropy weight and comprehensive index method," The Practice and Understanding of Mathematics, vol. 43, no. 13, pp. 50-57, 2015.

[15] K. Mori and A. Christodoulou, "Review of sustainability indices and indicators: towards a new city sustainability index (CSI)," Environmental Impact Assessment Review, vol. 32, no. 1, pp. 94-106, 2012.

[16] K. Mori, T. Fujii, T. Yamashita, Y. Mimura, Y. Uchiyama, and K. Hayashi, "Visualization of a city sustainability index (CSI): towards transdisciplinary approaches involving multiple stakeholders," Sustainability, vol. 7, no. 9, pp. 12402-12424, 2015.

[17] Y. A. Syed, P. Georgakis, and C. Nwagboso, "Fuzzy logicbased built environment impact assessment for urban regeneration simulation," in Proceedings of the 2009 2nd International Conference in Visualisation (IEEE), Barcelona, Spain, 2009.

[18] X. Wang, Research on Urban Design Methods for Waterfront Areas in Mountainous Cities under the Thought of Organic Cities: A Case Study of Chongqing's Main City Waterfront Location, Chongqing University, Chongqing, China, 2016. 